\title{
Nurses on the Frontline Against the COVID-19 Pandemic: Experience from a Tertiary Referral Cancer Center
}

\author{
Sindhu Nair ${ }^{1}$ Nishu Singh Goel ${ }^{2} \quad$ Swapna Joshi ${ }^{1} \quad$ Carmine Lasarado $^{1}$ Shweta Ghag ${ }^{1}$ \\ Chhaya Dhanve $^{1}$ Reena Nair ${ }^{1}$ Bharti Veer ${ }^{1}$ Rashmi Methry ${ }^{1}$
}

${ }^{1}$ Department of Nursing, Tata Memorial Centre, Homi Bhabha National Institute, Mumbai, Maharashtra, India

2 KEVAT-TMC Patient Navigation Program, Tata Memorial Centre, Homi Bhabha National Institute, Mumbai, Maharashtra, India

Ind J Med Paediatr Oncol 2022;43:24-28.

\section{Background}

Coronavirus disease 2019 (COVID-19) was first reported from Wuhan, China in late December 2019 and was declared a global pandemic by the World Health Organization (WHO) on March 11, 2020. As on November 23, 2021, the COVID-19 pandemic has infected more than 256 million individuals globally, leading to 5 million deaths and has disrupted health care systems across the world. ${ }^{1}$ Patients with cancer need prioritization of care, even during a pandemic. To continue cancer care in the face of the pandemic, cancer centers needed to evolve pathways to enable early identification of those with potential infections, and their high-risk contacts, and segregate them from the other noninfected patients. This was essential to contain the infection as well as minimize the risk of exposure to patients and the staff.

Nursing staff, who have always been key frontline health care workers, have been instrumental in the COVID-19 response worldwide, often at the cost of their own physical and emotional well-being. ${ }^{2,3}$ The Tata Memorial Hospital (TMH) is a grant-in-aid institution under the Department of Atomic Energy, Government of India and a tertiary referral cancer hospital. Early in the pandemic, the hospital was designated by the government as a COVID-19 hospital for all patients with cancer. There is lack of data from an oncology center regarding the impact of the double burden of COVID-19 and cancer on nursing staff in the face of lockdown, manpower shortage, and personal challenges. In this article, we describe the experience of nurses at the TMH
Address for correspondence Sindhu Nair, MSc, MA, Department of Nursing, Tata Memorial Centre, Homi Bhabha National Institute, Mumbai, Maharashtra 400012, India (e-mail: nairss@tmc.gov.in).

who were at the forefront of the fight against COVID-19, ably supported by others such as patient navigators, clinical research staff, and administrators.

\section{The Tata Memorial Hospital Experience}

India reported its first case of COVID-19 on January 27, 2020, in Kerala, and the first patient in Mumbai was diagnosed on March 9, 2020. The TMH began preparations to deal with the pandemic in early March 2020. Several policies were implemented in the hospital to mitigate the risk to staff, cancer patients, and caregivers to ensure effective hospital functioning. The initial preparations included creation of a core action group to review evidence, create and update standard operating procedures (SOPs), and oversee daily operations. Nursing staff were an integral part of this action group and expanded their services beyond the routine. The action group communicated via a WhatsApp group, and key members of the action group met on a daily basis to review the unfolding situation. The following areas were recognized for urgent action:

- Screening patients and staff to identify those likely to be infected

- Setting up of a fever clinic and facility for testing for COVID-19

- Creation of isolation wards and stepping up the infection prevention control measures
DOI https://doi.org/ $10.1055 / \mathrm{s}-0042-1743127$. ISSN 0971-5851. (c) 2022. Indian Society of Medical and Paediatric Oncology. All rights reserved.

This is an open access article published by Thieme under the terms of the Creative Commons Attribution-NonDerivative-NonCommercial-License, permitting copying and reproduction so long as the original work is given appropriate credit. Contents may not be used for commercial purposes, or adapted, remixed, transformed or built upon. (https://creativecommons.org/ licenses/by-nc-nd/4.0/)

Thieme Medical and Scientific Publishers Pvt. Ltd., A-12, 2nd Floor, Sector 2, Noida-201301 UP, India 
- Education, training, and audits

- Management of infected cases and their contacts

- Contact tracing.

Subcommittees of the core action group were created to look into each of these aspects. A team of medical staff constantly updated the SOPs based on evolving evidence.

Subsequently, when TMH became a COVID-19 vaccination center in March 2021, nursing staff took on the additional responsibility of managing the vaccination center.

\section{Screening of Patients and Staff}

One of the measures adopted since March 2020 was screening of patients and accompanying persons at hospital entry points, and thermal screening for hospital staff. The screening was undertaken by the frontline health care personnel, which involved mainly the KEVAT (patient navigators), and the staff deputed from various departments of the hospital such as preventive oncology, clinical research, nursing, and administration. The identified frontline personnel were rendered intensive training on administering a COVID-19 questionnaire and use of thermal screening to identify high-risk individuals, thus segregating the COVID-19 suspects from others. Those who were identified as high risk- having history of travel, symptoms, high body temperature-were referred to a dedicated "fever clinic" for further evaluation. Others who were screened negative at the entrance were directed to access regular hospital services. Entry access into the hospital was limited to only one person accompanying the patient, extendable to two if the patient was on a wheelchair. The frontline staff were trained to safeguard themselves from the infection. This training included appropriate use of personal protective equipment (PPE), social distancing, and hand hygiene.

\section{Setting up of a Fever Clinic and Facility for Testing for COVID-19}

A section of the main part of the hospital near the entrance was converted to a "fever clinic" where screened individuals identified as high risk could be evaluated further.

Facilities for collecting swabs for reverse transcription polymerase chain reaction testing, waiting areas for those awaiting the results of the swab, and separate entrance and exit pathways to avoid mingling between suspects and other staff and patients were also set up. Nursing staff assisted doctors in the fever OPD for sample collection.

\section{Education, Training, and Audits}

We recognized that hospital staff, in addition to fulfilling their professional roles, were also individuals who were concerned about their own safety and that of their families. They were also key members of the community who could play a role in spreading the right knowledge about COVID-19. Therefore, we started the process of training hospital staff on various aspects of COVID-19. A group of nursing staff under- went the initial training and then acted as trainers for the other individuals. These training modules were conducted in small batches and included generating awareness about the symptoms of COVID-19, and precautions to be followed by all (masking, physical distancing, cough etiquette, and hand hygiene). In addition, they were taught about infection control practices to be followed in the wards and critical care areas such as appropriate use of PPE, measures to be followed while caring for those with COVID-19, and management of waste disposal. Over the period of 2 months (March and April 2020), 919 nursing staff and 1,322 ancillary staff received this training. In addition, 575 nursing staff underwent online training in COVID-19 protocols to be followed. Periodic audits were performed to assess retention of information and need for retraining. The nursing department also undertook the task of training housekeeping personnel on infection prevention and control measures.

\section{Management of Infected Cases and Their Contacts}

\section{Setting up of an Isolation Ward}

The first step was to identify a separate ward which was converted into an isolation ward to accommodate COVID19 cases. Since the hospital had limited space, and needed to continue cancer care, it was decided that only those with symptomatic COVID-19 or risk factors would be accommodated in the hospital premises. A separate facility near the hospital was identified as an isolation facility for those with asymptomatic or mild COVID-19, who did not have facilities for home isolation. Within the hospital's existing facilities, new areas were set up by reorganizing various inpatient and outpatient areas to create segregated space for COVID-19 care. An intensive care unit (ICU) facility was created for the patients who needed ventilatory support and close monitoring. A separate pathway for transport of patients with COVID-19 was identified, rooms for donning and doffing of PPE were set up, and several trial runs were performed much before we had our first patient to ensure that we were well prepared to safely manage patients with COVID-19.

\section{Tracing of Contacts}

Identifying high-risk contacts of infected individuals is important to minimize the extent of transmission. Nursing staff created a group which had individuals trained in SOPs of contact tracing. The group efficiently conducted interviews of all possible contacts to identify those at high risk. Those who were considered high risk were quarantined at suitable locations. Initially, the quarantine facility was set up in the hospital itself, while later on, other premises such as hotels and hostels were used as quarantine facilities.

\section{Testing/Retesting of Cases and Contacts}

Nursing staff prepared databases of those in isolation and quarantine and arranged for them to be tested at recommended intervals prior to termination of isolation or quarantine. Since the guidelines for duration of isolation/quarantine 
and time point for retesting were constantly evolving, the nurses adapted their SOPs accordingly.

\section{Arranging Minimum Supplies for Those in Isolation or Quarantine}

Recognizing that individuals who were isolated or quarantined may not have had time to make preparations, nursing staff partnered with nongovernmental organizations to prepare and provide basic hygiene kits to these individuals to tide them over the initial period.

\section{Changes in Working Pattern}

Since COVID-19 management and cancer care had to be performed with the same number of nursing staff, several changes were made to the working pattern. First, staff who were high risk for COVID-19 (pregnant, multiple comorbidities, and immunosuppressed) were given medical leave during the peak of the pandemic. A proportion of staff (around 40\%) from medical and surgical areas were retrained in critical care skills and redeployed in ICUs. Duty shifts in COVID-19 areas were drawn up to include staggered shifts so that the duration of time spent in PPE was minimized. The "buddy" concept was brought in where staff on duty alternately spent a part of their shift time attending to tasks which did not require direct patient contact or the use of PPE. At periodic intervals, staff were rotated from COVID-19 to nonCOVID-19 duty areas, to avoid fatigue. All attempts were made to ensure the physical and emotional well-being of the nursing staff. - Fig. 1 outlines the nursing staffing pattern during the pandemic.

\section{Nursing Education}

Nursing education was also affected by the pandemic. TMH runs courses in oncology nursing such as diploma in oncology nursing and masters in oncology nursing. In the initial period of the pandemic, all the students were utilized in the clinical area for patient care. Diploma oncology students were part of the screening team and MSc nursing students were part of the contact tracing team. Online mode of training was utilized for lectures to reduce the impact of pandemic on their learning.

\section{Vaccination}

TMH became a COVID-19 vaccination center in March 2021. Nursing staff allocated to this center were sent to the COVID19 vaccination center at the nearby municipal hospital for training. The nursing supervisor also visited other hospitals to plan facilities for the COVID-19 vaccination center at TMH. The responsibilities of nursing staff at the vaccination center involved retrieving the vaccines from the central storage area, maintaining the cold chain, administration of vaccine, keeping a critical care unit ready for any emergencies, and maintaining a record of beneficiaries.

\section{Challenges and the Way Forward}

On April 12, 2020, the first case of COVID-19 was diagnosed in TMH. Since then, more than 2,500 patients and 1,000 staff have tested positive for COVID-19 (until October 2021) (-Fig. 2). Around 200 nursing staff have tested positive and 100 have been quarantined until then. Some nursing

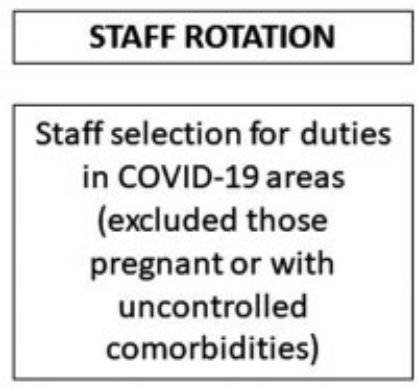

Nursing staff from non-
critical units like OPD and
diagnostics were
deployed to critical units
like ICU and isolation
ward.

Transfer of staff to non COVID-19 area after 30 working days in COVID19 area
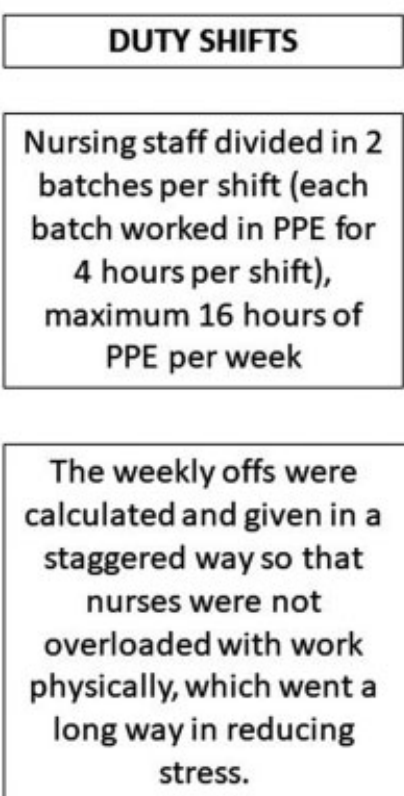

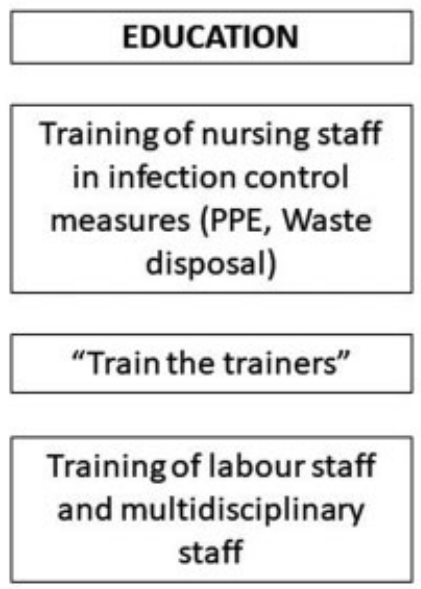

Training of labour staff

staff

Fig. 1 Nursing staffing model during the pandemic. COVID-19, coronavirus disease 2019; ICU, intensive care unit; PPE, personal protective equipment. 


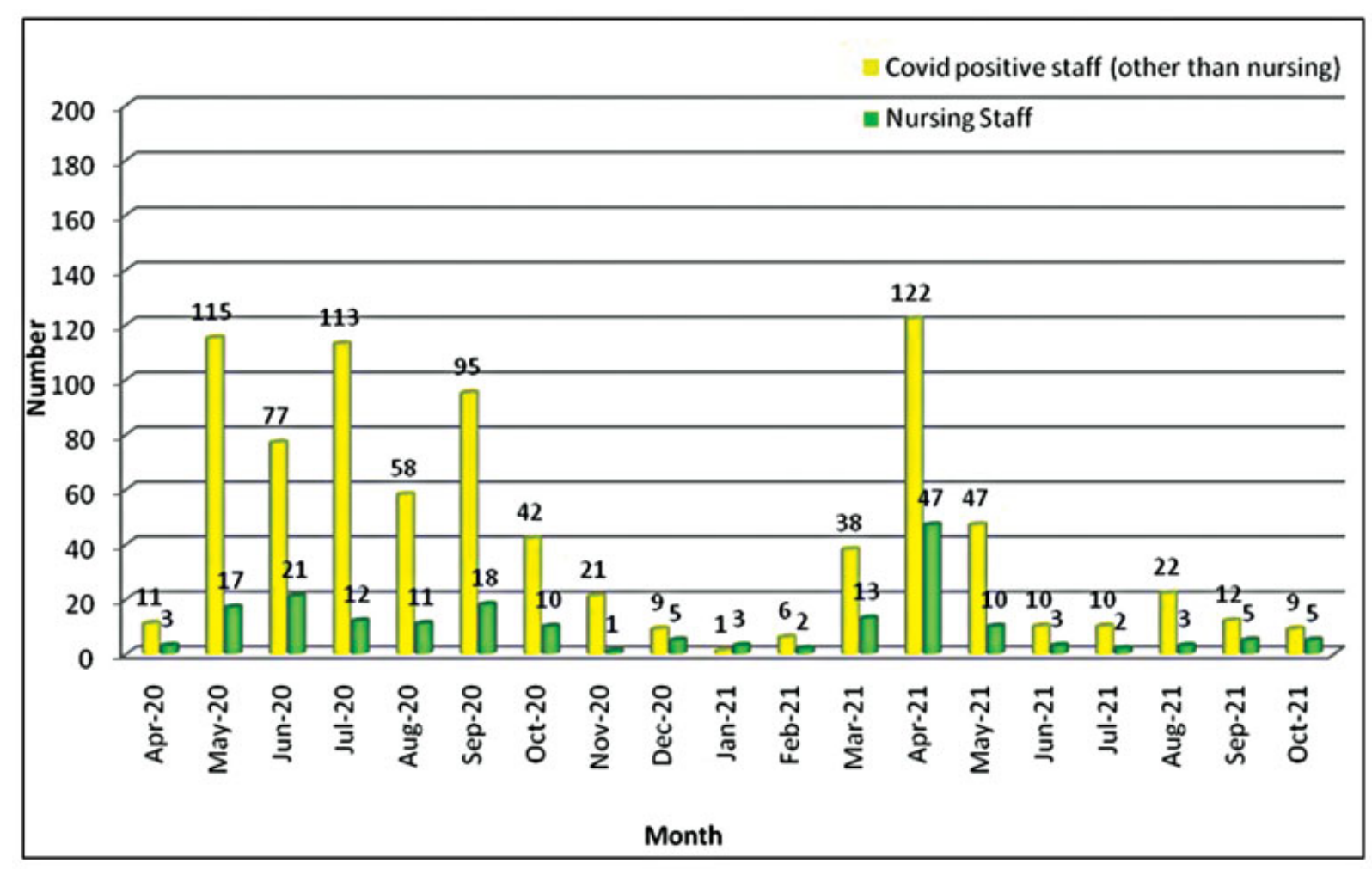

Fig. 2 Number of coronavirus disease 2019-positive staff in the Tata Memorial Hospital.

staff have reported personal challenges such as social stigma from neighbors and family or the need to stay away from home to avoid infecting family members. Several staff have dealt with mental health issues such as anxiety and burnout. Most of the nurses who used PPE for prolonged periods reported various types of injuries and discomfort. With the pandemic extending for almost 18 months, fatigue has set in, and there is the need to constantly reinforce and retrain appropriate behavioral measures.

As COVID-19 cases plateau and we adjust to the new "normal," cancer care has returned to its usual volumes. The role of the TMH nursing team in dealing with the pandemic has been acknowledged by the hospital administration as one of the most crucial aspects of the overall response. Nurses presented their observations and learnings in hospital meetings and in national webinars, to allow others to benefit from their experience.

\section{Discussion}

The WHO estimates that 80,000 to 180,000 health care workers could have died from COVID-19 in the period between January 2020 and May 2021. ${ }^{4}$ Several of them are likely to have been nursing staff. Bandyopadhyay et al looked at COVID-19 infections and deaths among health care workers and found that among those infected, nurses constituted the largest proportion (38\%). ${ }^{3}$

The impact of the pandemic on the mental health of nurses has also been well documented, with several studies reporting anxiety, depression, stress, burnout among nurs- ing staff. ${ }^{5}$ A systematic review and meta-analysis looking at psychological distress among health care providers during COVID-19 in Asia found that more than one-third of health care providers suffered from anxiety and depression, the likelihood being higher with female gender and nurses. ${ }^{6}$ Lack of human and physical resources and the number of colleagues infected with COVID-19 were the strongest predictors of stress, anxiety, and depression among nurses. ${ }^{7}$ Sharma et al surveyed nurses at the frontline in government hospitals across India and found that 12 to $14 \%$ of them reported anxiety and depression among frontline nurses. ${ }^{8}$ Nurses have been at the receiving end of bullying and social stigma due to the perception that they are carriers of COVID-19. ${ }^{5}$ In a study among health care workers in India, Radhakrishnan et al found that $70 \%$ of nurses reported a stigmatizing experience during COVID-19, and that being a nurse and working in a clinical area were more likely to worsen this experience. ${ }^{9}$ Most of these reports are from the initial period of the pandemic where transmission was less understood and fear was high. It is to be hoped that several months into the pandemic, and recognizing the vital role that health care workers have played, such problems and stigma do not exist anymore.

The use of PPE is associated with problems such as pressure injuries, headaches, sweating, disturbances of vision, and difficulty in breathing. It has been estimated that three out of four individuals who use PPE are likely to have adverse events related to skin. ${ }^{10}$ Nurses, due to their long-duration shifts and fixed postings in COVID-19 wards, are more likely to experience such problems. 
Wearing PPE for longer than 4 hours has been identified as a significant factor for adverse events, and limiting the duration of PPE to less than 4 hours could be the solution. $^{11}$

The training of nursing students during the pandemic has also been affected, with the switch to online classes. Their clinical experience has been limited by frequent postings in COVID-19 wards and ICU. In a systematic review of studies from around the world, Mulyadi et al found that nursing students had high rates of stress, anxiety, fear, depression, and sleep disturbances during the pandemic. ${ }^{12}$ In another study, more than $90 \%$ of nursing students reported a lack of proficiency with the use of internet and online teaching methods. ${ }^{13}$ It is essential that their skills and confidence should be reinforced in the remaining part of their training schedule.

Nurses, whether posted in wards, operating rooms, or ICUs, have always been a part of a multidisciplinary team and recognize the importance of teamwork through collaboration and good communication. During the pandemic, the nursing team at $\mathrm{TMH}$ has taken teamwork to a new high and worked in close partnership with several other departments -patient navigation teams, administration, medical staff, clinical research staff, and many others, to ensure smooth functioning and better outcomes. Their combined efforts have proved that it is possible to continue routine medical care while simultaneously managing the COVID-19 situation. For conditions such as cancer, where any delays in diagnosis or treatment affects patient outcomes, this learning is important.

\section{Conclusion}

The WHO had designated the year 2020 as the "year of the nurse and midwife," in honor of the 200th birth anniversary of Florence Nightingale. It is appropriate that in this year, nurses have been the champions in the battle against COVID19. However, in the course of this battle, nursing staff have faced several challenges which need to be addressed to ensure their continued well-being. Hospital administrations should recognize the critical role that nurses play in crisis situations and empower them while they deliver important aspects of the overall response.
Conflict of Interest

None declared.

\section{References}

1 WHO. COVID-19 dashboard. World Health Organization. Accessed November 23, 2021 at: https://covid19.who.int/

2 Varghese A, George G, Kondaguli SV, Naser AY, Khakha DC, Chatterji R. Decline in the mental health of nurses across the globe during COVID-19: a systematic review and meta-analysis. J Glob Health 2021;11:05009

3 Bandyopadhyay S, Baticulon RE, Kadhum M, et al. Infection and mortality of healthcare workers worldwide from COVID-19: a systematic review. BMJ Glob Health 2020;5(12):e003097

4 WHO and partners call for action to better protect health and care workers from COVID-19. World Health Organization. Accessed February 3, 2022 at: https://www.who.int/news/item/21-10-2021who-and-partners-call-for-action-to-better-protect-health-and-careworkers-from-covid-19\#: :text=In\%20a\%20Joint\%20Statement\%20 issued,among\%20health\%20and\%20care\%20workers

5 Barrett D, Heale R. COVID-19: reflections on its impact on nursing. Evid Based Nurs 2021;24(04):112-113

6 Chen SC, Lai YH, Tsay SL. Nursing perspectives on the impacts of COVID-19. J Nurs Res 2020;28(03):e85

7 Baraka AAE, Ramadan FH, Hassan EA. Predictors of critical care nurses' stress, anxiety, and depression in response to COVID-19 pandemic. Nurs Crit Care 2021 (e-pub ahead of print). Doi: $10.1111 /$ nicc. 12708

8 Sharma SK, Mudgal SK, Thakur K, Parihar A, Chundawat DS, Joshi J. Anxiety, depression and quality of life (QOL) related to COVID-19 among frontline health care professionals: a multicentric crosssectional survey. J Family Med Prim Care 2021;10(03):1383-1389

9 Radhakrishnan RV, Jain M, Mohanty CR, et al. The perceived social stigma, self-esteem, and its determinants among the health care professionals working in India during COVID 19 pandemic. Med J Armed Forces India 2021;77(Suppl 2):S450-S458

10 Montero-Vilchez T, Cuenca-Barrales C, Martinez-Lopez A, MolinaLeyva A, Arias-Santiago S. Skin adverse events related to personal protective equipment: a systematic review and meta-analysis. J Eur Acad Dermatol Venereol 2021;35(10):1994-2006

11 Atay S, Cura ŞÜ Problems encountered by nurses due to the use of personal protective equipment during the coronavirus pandemic: results of a survey. Wound Manag Prev 2020;66(10):12-16

12 Mulyadi M, Tonapa SI, Luneto S, Lin WT, Lee BO. Prevalence of mental health problems and sleep disturbances in nursing students during the COVID-19 pandemic: a systematic review and meta-analysis. Nurse Educ Pract 2021;57:103228

13 Singh HK, Joshi A, Malepati RN, et al. A survey of e-learning methods in nursing and medical education during COVID-19 pandemic in India. Nurse Educ Today 2021;99:104796 